ISSN 1855-3966 (printed edn.), ISSN 1855-3974 (electronic edn.)

\author{
ARS MATHEMATICA CONTEMPORANEA 19 (2020) 277-295 \\ https://doi.org/10.26493/1855-3974.2348.f42 \\ (Also available at http://amc-journal.eu)
}

\title{
The Cayley isomorphism property for the group $C_{2}^{5} \times C_{p}$
}

\author{
Grigory Ryabov * \\ Sobolev Institute of Mathematics, \\ 4 Acad. Koptyug avenue, 630090, Novosibirsk, Russia, and \\ Novosibirsk State University, 1 Pirogova st., 630090, Novosibirsk, Russia
}

Received 28 May 2020, accepted 29 July 2020, published online 17 November 2020

\begin{abstract}
A finite group $G$ is called a DCI-group if two Cayley digraphs over $G$ are isomorphic if and only if their connection sets are conjugate by a group automorphism. We prove that the group $C_{2}^{5} \times C_{p}$, where $p$ is a prime, is a DCI-group if and only if $p \neq 2$. Together with the previously obtained results, this implies that a group $G$ of order $32 p$, where $p$ is a prime, is a DCI-group if and only if $p \neq 2$ and $G \cong C_{2}^{5} \times C_{p}$.
\end{abstract}

Keywords: Isomorphisms, DCI-groups, Schur rings.

Math. Subj. Class. (2020): 05C25, 05C60, $20 B 25$

\section{Introduction}

Let $G$ be a finite group and $S \subseteq G$. The Cayley digraph Cay $(G, S)$ over $G$ with connection set $S$ is defined to be the digraph with vertex set $G$ and arc set $\{(g, s g): g \in G, s \in S\}$. Two Cayley digraphs over $G$ are called Cayley isomorphic if there exists an isomorphism between them which is also an automorphism of $G$. Clearly, two Cayley isomorphic Cayley digraphs are isomorphic. The converse statement is not true in general (see [3, 10]). A subset $S \subseteq G$ is called a CI-subset if for each $T \subseteq G$ the Cayley digraphs $\operatorname{Cay}(G, S)$ and $\operatorname{Cay}(G, T)$ are isomorphic if and only if they are Cayley isomorphic. A finite group $G$ is called a DCI-group (CI-group, respectively) if each subset of $G$ (each inverse-closed subset of $G$, respectively) is a CI-subset.

* The work is supported by Mathematical Center in Akademgorodok under agreement No. 075-15-2019-1613 with the Ministry of Science and Higher Education of the Russian Federation.

The author would like to thank Prof. István Kovács for the fruitful discussions on the subject matters, Prof. Pablo Spiga and the anonymous referee for valuable comments which help to improve the text significantly.

E-mail address: gric2ryabov@gmail.com (Grigory Ryabov) 
The investigation of DCI-groups was initiated by Ádám [1] who conjectured, in our terms, that every cyclic group is a DCI-group. This conjecture was disproved by Elspas and Turner in [10]. The problem of determining of finite DCI- and CI-groups was suggested by Babai and Frankl in [5]. For more information on DCI- and CI-groups we refer the readers to the survey paper [21].

In this paper we are interested in abelian DCI-groups. The cyclic group of order $n$ is denoted by $C_{n}$. Elspas and Turner [10] and independently Djoković [8] proved that every cyclic group of prime order is a DCI-group. The fact that $C_{p q}$ is a DCI-group for distinct primes $p$ and $q$ was proved by Alspach and Parsons in [3] and independently by Klin and Pöschel in [17]. The complete classification of cyclic DCI-groups was obtained by Muzychuk in $[23,24]$. He proved that a cyclic group of order $n$ is a DCI-group if and only if $n=k$ or $n=2 k$, where $k$ is square-free.

Denote the class of all finite abelian groups where every Sylow subgroup is elementary abelian by $\mathcal{E}$. From [18, Theorem 1.1] it follows that every DCI-group is the coprime product (i.e. the direct product of groups of coprime orders) of groups from the following list:

$$
C_{p}^{k}, C_{4}, Q_{8}, A_{4}, H \rtimes\langle z\rangle,
$$

where $p$ is a prime, $H$ is a group of odd order from $\mathcal{E},|z| \in\{2,4\}$, and $h^{z}=h^{-1}$ for every $h \in H$. One can check that the class of DCI-groups is closed under taking subgroups. So one of the crucial steps towards the classification of all DCI-groups is to determine which groups from $\mathcal{E}$ are DCI.

The following non-cyclic groups from $\mathcal{E}$ are DCI-groups ( $p$ and $q$ are assumed to be distinct primes): $C_{p}^{2}[2,14] ; C_{p}^{3}$ [2, 9]; $C_{2}^{4}, C_{2}^{5}$ [7]; $C_{p}^{4}$, where $p$ is odd [15] (a proof for $C_{p}^{4}$ with no condition on $p$ was given in [22]); $C_{p}^{5}$, where $p$ is odd [13]; $C_{p}^{2} \times C_{q}$ [18]; $C_{p}^{3} \times C_{q}$ [27]; $C_{p}^{4} \times C_{q}$ [20]. The smallest example of a non-DCI-group from $\mathcal{E}$ was found by Nowitz [28]. He proved that $C_{2}^{6}$ is non-DCI. This implies that $C_{2}^{n}$ is non-DCI for every $n \geq 6$. Also $C_{3}^{n}$ is non-DCI for every $n \geq 8$ [33] and $C_{p}^{n}$ is non-DCI for every prime $p$ and $n \geq 2 p+3$ [32].

In this paper we find a new infinite family of DCI-groups from $\mathcal{E}$ which are close to the smallest non-DCI-group from $\mathcal{E}$. The main result of the paper can be formulated as follows.

Theorem 1.1. Let $p$ be a prime. Then the group $C_{2}^{5} \times C_{p}$ is a DCI-group if and only if $p \neq 2$.

Theorem 1.1 extends the results obtained in [18, 20, 27] which imply that the group $C_{p}^{k} \times C_{q}$ is a DCI-group whenever $p$ and $q$ are distinct primes and $k \leq 4$. Note that the "only if" part of Theorem 1.1, in fact, was proved by Nowitz in [28]. The next corollary immediately follows from [18, Theorem 1.1] and Theorem 1.1.

Corollary 1.2. Let $p$ be a prime. Then a group $G$ of order $32 p$ is a DCI-group if and only if $p \neq 2$ and $G \cong C_{2}^{5} \times C_{p}$.

To prove Theorem 1.1, we use the $S$-ring approach. An $S$-ring over a group $G$ is a subring of the group ring $\mathbb{Z} G$ which is a free $\mathbb{Z}$-module spanned by a special partition of $G$. If every $S$-ring from a certain family of $S$-rings over $G$ is a CI- $S$-ring then $G$ is a DCI-group (see Section 4). The definition of an $S$-ring goes back to Schur [31] and Wielandt [34]. The usage of $S$-rings in the investigation of DCI-groups was proposed by 
Klin and Pöschel [17]. Most recent results on DCI-groups were obtained using $S$-rings (see [15, 18, 19, 20, 27]).

The text of the paper is organized in the following way. In Section 2 we provide definitions and basic facts concerned with $S$-rings. Section 3 contains a necessary information on isomorphisms of $S$-rings. In Section 4 we discuss CI- $S$-rings and their relation with DCI-groups. We also prove in this section a sufficient condition of CI-property for $S$-rings (Lemma 4.4). Section 5 is devoted to the generalized wreath and star products of $S$-rings. Here we deduce from previously obtained results two sufficient conditions for the generalized wreath product of $S$-rings to be a CI-S-ring (Lemma 5.5 and Lemma 5.8). Section 6 and 7 are concerned with $p$-S-rings and $S$-rings over a group of order $p k$, where $p$ is a prime and $\operatorname{GCD}(p, k)=1$, (so-called non-powerful order) respectively. In Section 8 we provide properties of $S$-rings over the groups $C_{2}^{n}, n \leq 5$, and prove that all $S$-rings over these groups are CI. The material of this section is based on computational results obtained with the help of the GAP package COCO2P [16]. Finally, in Section 9 we prove Theorem 1.1.

Notation. Let $G$ be a finite group and $X \subseteq G$. The element $\sum_{x \in X} x$ of the group ring $\mathbb{Z} G$ is denoted by $\underline{X}$.

The set $\left\{x^{-1}: x \in X\right\}$ is denoted by $X^{-1}$.

The subgroup of $G$ generated by $X$ is denoted by $\langle X\rangle$; we also set $\operatorname{rad}(X)=\{g \in G$ : $g X=X g=X\}$.

Given a set $X \subseteq G$ the set $\{(g, x g): x \in X, g \in G\}$ of arcs of the Cayley digraph $\operatorname{Cay}(G, X)$ is denoted by $A(X)$.

The group of all permutations of $G$ is denoted by $\operatorname{Sym}(G)$.

The subgroup of $\operatorname{Sym}(G)$ consisting of all right translations of $G$ is denoted by $G_{\text {right }}$.

The set $\left\{K \leq \operatorname{Sym}(G): K \geq G_{\text {right }}\right\}$ is denoted by $\operatorname{Sup}\left(G_{\text {right }}\right)$.

For a set $\Delta \subseteq \operatorname{Sym}(G)$ and a section $S=U / L$ of $G$ we set $\Delta^{S}=\left\{f^{S}: f \in \Delta, S^{f}=\right.$ $S$, where $S^{f}=S$ means that $f$ permutes the $L$-cosets in $U$ and $f^{S}$ denotes the bijection of $S$ induced by $f$.

If $K \leq \operatorname{Sym}(\Omega)$ and $\alpha \in \Omega$ then the stabilizer of $\alpha$ in $K$ and the set of all orbits of $K$ on $\Omega$ are denoted by $K_{\alpha}$ and $\operatorname{Orb}(K, \Omega)$ respectively.

If $H \leq G$ then the normalizer of $H$ in $G$ is denoted by $N_{G}(H)$.

The cyclic group of order $n$ is denoted by $C_{n}$.

The class of all finite abelian groups where every Sylow subgroup is elementary abelian is denoted by $\mathcal{E}$.

\section{$2 S$-rings}

In this section we give a background of $S$-rings. In general, we follow [20], where the most part of the material is contained. For more information on $S$-rings we refer the readers to $[6,25]$.

Let $G$ be a finite group and $\mathbb{Z} G$ the integer group ring. Denote the identity element of $G$ by $e$. A subring $\mathcal{A} \subseteq \mathbb{Z} G$ is called an $S$-ring (a Schur ring) over $G$ if there exists a partition $\mathcal{S}(\mathcal{A})$ of $G$ such that:

(1) $\{e\} \in \mathcal{S}(\mathcal{A})$,

(2) if $X \in \mathcal{S}(\mathcal{A})$ then $X^{-1} \in \mathcal{S}(\mathcal{A})$,

(3) $\mathcal{A}=\operatorname{Span}_{\mathbb{Z}}\{\underline{X}: X \in \mathcal{S}(\mathcal{A})\}$. 
The elements of $\mathcal{S}(\mathcal{A})$ are called the basic sets of $\mathcal{A}$ and the number $\operatorname{rk}(\mathcal{A})=|\mathcal{S}(\mathcal{A})|$ is called the rank of $\mathcal{A}$. If $X, Y \in \mathcal{S}(\mathcal{A})$ then $X Y \in \mathcal{S}(\mathcal{A})$ whenever $|X|=1$ or $|Y|=1$.

Let $\mathcal{A}$ be an $S$-ring over a group $G$. A set $X \subseteq G$ is called an $\mathcal{A}$-set if $X \in \mathcal{A}$. A subgroup $H \leq G$ is called an $\mathcal{A}$-subgroup if $H$ is an $\mathcal{A}$-set. From the definition it follows that the intersection of $\mathcal{A}$-subgroups is also an $\mathcal{A}$-subgroup. One can check that for each $\mathcal{A}$-set $X$ the groups $\langle X\rangle$ and $\operatorname{rad}(X)$ are $\mathcal{A}$-subgroups. By the thin radical of $\mathcal{A}$ we mean the set defined as

$$
\mathbf{O}_{\theta}(\mathcal{A})=\{x \in G:\{x\} \in \mathcal{S}(\mathcal{A})\}
$$

It is easy to see that $\mathbf{O}_{\theta}(\mathcal{A})$ is an $\mathcal{A}$-subgroup.

Lemma 2.1 ([11, Lemma 2.1]). Let $\mathcal{A}$ be an $S$-ring over a group $G, H$ an $\mathcal{A}$-subgroup of $G$, and $X \in \mathcal{S}(\mathcal{A})$. Then the number $|X \cap H x|$ does not depend on $x \in X$.

Let $L \unlhd U \leq G$. A section $U / L$ is called an $\mathcal{A}$-section if $U$ and $L$ are $\mathcal{A}$-subgroups. If $S=U / L$ is an $\mathcal{A}$-section then the module

$$
\mathcal{A}_{S}=\operatorname{Span}_{\mathbb{Z}}\left\{\underline{X}^{\pi}: X \in \mathcal{S}(\mathcal{A}), X \subseteq U\right\}
$$

where $\pi: U \rightarrow U / L$ is the canonical epimorphism, is an $S$-ring over $S$.

\section{Isomorphisms and schurity}

Let $\mathcal{A}$ and $\mathcal{A}^{\prime}$ be $S$-rings over groups $G$ and $G^{\prime}$ respectively. A bijection $f: G \rightarrow G^{\prime}$ is called an isomorphism from $\mathcal{A}$ to $\mathcal{A}^{\prime}$ if

$$
\left\{A(X)^{f}: X \in \mathcal{S}(\mathcal{A})\right\}=\left\{A\left(X^{\prime}\right): X^{\prime} \in \mathcal{S}\left(\mathcal{A}^{\prime}\right)\right\}
$$

where $A(X)^{f}=\left\{\left(g^{f}, h^{f}\right):(g, h) \in A(X)\right\}$. If there exists an isomorphism from $\mathcal{A}$ to $\mathcal{A}^{\prime}$ then we say that $\mathcal{A}$ and $\mathcal{A}^{\prime}$ are isomorphic and write $\mathcal{A} \cong \mathcal{A}^{\prime}$.

The group of all isomorphisms from $\mathcal{A}$ onto itself contains a normal subgroup

$$
\left\{f \in \operatorname{Sym}(G): A(X)^{f}=A(X) \text { for every } X \in \mathcal{S}(\mathcal{A})\right\}
$$

called the automorphism group of $\mathcal{A}$ and denoted by $\operatorname{Aut}(\mathcal{A})$. The definition implies that $G_{\text {right }} \leq \operatorname{Aut}(\mathcal{A})$. The $S$-ring $\mathcal{A}$ is called normal if $G_{\text {right }}$ is normal in $\operatorname{Aut}(\mathcal{A})$. One can verify that if $S$ is an $\mathcal{A}$-section then $\operatorname{Aut}(\mathcal{A})^{S} \leq \operatorname{Aut}\left(\mathcal{A}_{S}\right)$. Denote the group $\operatorname{Aut}(\mathcal{A}) \cap$ $\operatorname{Aut}(G)$ by $\operatorname{Aut}_{G}(\mathcal{A})$. It easy to check that if $S$ is an $\mathcal{A}$-section then $\operatorname{Aut}_{G}(\mathcal{A})^{S} \leq$ $\operatorname{Aut}_{S}\left(\mathcal{A}_{S}\right)$. One can verify that

$$
\operatorname{Aut}_{G}(\mathcal{A})=\left(N_{\operatorname{Aut}(\mathcal{A})}\left(G_{\text {right }}\right)\right)_{e} .
$$

Let $K \in \operatorname{Sup}\left(G_{\text {right }}\right)$. Schur proved in [31] that the $\mathbb{Z}$-submodule

$$
V(K, G)=\operatorname{Span}_{\mathbb{Z}}\left\{\underline{X}: X \in \operatorname{Orb}\left(K_{e}, G\right)\right\},
$$

is an $S$-ring over $G$. An $S$-ring $\mathcal{A}$ over $G$ is called schurian if $\mathcal{A}=V(K, G)$ for some $K \in \operatorname{Sup}\left(G_{\text {right }}\right)$. One can verify that given $K_{1}, K_{2} \in \operatorname{Sup}\left(G_{\text {right }}\right)$,

$$
\text { if } K_{1} \leq K_{2} \text { then } V\left(K_{1}, G\right) \geq V\left(K_{2}, G\right)
$$


If $\mathcal{A}=V(K, G)$ for some $K \in \operatorname{Sup}\left(G_{\text {right }}\right)$ and $S$ is an $\mathcal{A}$-section then $\mathcal{A}_{S}=V\left(K^{S}, S\right)$. So if $\mathcal{A}$ is schurian then $\mathcal{A}_{S}$ is also schurian for every $\mathcal{A}$-section $S$. It can be checked that

$$
V(\operatorname{Aut}(\mathcal{A}), G) \geq \mathcal{A}
$$

and the equality is attained if and only if $\mathcal{A}$ is schurian.

An $S$-ring $\mathcal{A}$ over a group $G$ is defined to be cyclotomic if there exists $K \leq \operatorname{Aut}(G)$ such that $\mathcal{S}(\mathcal{A})=\operatorname{Orb}(K, G)$. In this case we write $\mathcal{A}=\operatorname{Cyc}(K, G)$. Obviously, $\mathcal{A}=$ $V\left(G_{\text {right }} K, G\right)$. So every cyclotomic $S$-ring is schurian. If $\mathcal{A}=\operatorname{Cyc}(K, G)$ for some $K \leq \operatorname{Aut}(G)$ and $S$ is an $\mathcal{A}$-section then $\mathcal{A}_{S}=\operatorname{Cyc}\left(K^{S}, S\right)$. Therefore if $\mathcal{A}$ is cyclotomic then $\mathcal{A}_{S}$ is also cyclotomic for every $\mathcal{A}$-section $S$.

Two permutation groups $K_{1}$ and $K_{2}$ on a set $\Omega$ are called 2-equivalent if $\operatorname{Orb}\left(K_{1}, \Omega^{2}\right)=$ $\operatorname{Orb}\left(K_{2}, \Omega^{2}\right)$ (here we assume that $K_{1}$ and $K_{2}$ act on $\Omega^{2}$ componentwise). In this case we write $K_{1} \approx_{2} K_{2}$. The relation $\approx_{2}$ is an equivalence relation on the set of all subgroups of $\operatorname{Sym}(\Omega)$. Every equivalence class has a unique maximal element with respect to inclusion. Given $K \leq \operatorname{Sym}(\Omega)$, this element is called the 2-closure of $K$ and denoted by $K^{(2)}$. If $\mathcal{A}=V(K, G)$ for some $K \in \operatorname{Sup}\left(G_{\text {right }}\right)$ then $K^{(2)}=\operatorname{Aut}(\mathcal{A})$. An $S$-ring $\mathcal{A}$ over $G$ is called 2-minimal if

$$
\left\{K \in \operatorname{Sup}\left(G_{\text {right }}\right): K \approx_{2} \operatorname{Aut}(\mathcal{A})\right\}=\{\operatorname{Aut}(\mathcal{A})\} .
$$

Two groups $K_{1}, K_{2} \leq \operatorname{Aut}(G)$ are said to be Cayley equivalent if $\operatorname{Orb}\left(K_{1}, G\right)=$ $\operatorname{Orb}\left(K_{2}, G\right)$. In this case we write $K_{1} \approx_{\text {Cay }} K_{2}$. If $\mathcal{A}=\operatorname{Cyc}(K, G)$ for some $K \leq$ $\operatorname{Aut}(G)$ then $\operatorname{Aut}_{G}(\mathcal{A})$ is the largest group which is Cayley equivalent to $K$. A cyclotomic $S$-ring $\mathcal{A}$ over $G$ is called Cayley minimal if

$$
\left\{K \leq \operatorname{Aut}(G): K \approx_{\text {Cay }} \operatorname{Aut}_{G}(\mathcal{A})\right\}=\left\{\operatorname{Aut}_{G}(\mathcal{A})\right\} .
$$

It is easy to see that $\mathbb{Z} G$ is 2 -minimal and Cayley minimal.

\section{CI-S-rings}

Let $\mathcal{A}$ be an $S$-ring over a group $G$. Put

$$
\operatorname{Iso}(\mathcal{A})=\{f \in \operatorname{Sym}(G): f \text { is an isomorphism from } \mathcal{A} \text { onto an } S \text {-ring over } G\} .
$$

One can see that $\operatorname{Aut}(\mathcal{A}) \operatorname{Aut}(G) \subseteq \operatorname{Iso}(\mathcal{A})$. However, the converse statement does not hold in general. The $S$-ring $\mathcal{A}$ is defined to be a CI-S-ring if $\operatorname{Aut}(\mathcal{A}) \operatorname{Aut}(G)=\operatorname{Iso}(\mathcal{A})$. It is easy to check that $\mathbb{Z} G$ and the $S$-ring of rank 2 over $G$ are CI- $S$-rings.

Put

$$
\operatorname{Sup}_{2}\left(G_{\text {right }}\right)=\left\{K \in \operatorname{Sup}\left(G_{\text {right }}\right): K^{(2)}=K\right\} .
$$

The group $M \leq \operatorname{Sym}(G)$ is said to be $G$-regular if $M$ is regular and isomorphic to $G$. Following [15], we say that a group $K \in \operatorname{Sup}\left(G_{\text {right }}\right)$ is $G$-transjugate if every $G$-regular subgroup of $K$ is $K$-conjugate to $G_{\text {right }}$. Babai proved in [4] the statement which can be formulated in our terms as follows: a set $S \subseteq G$ is a CI-subset if and only if the group $\operatorname{Aut}(\operatorname{Cay}(G, S))$ is $G$-transjugate. The next lemma provides a similar criterion for a schurian $S$-ring to be CI.

Lemma 4.1. Let $K \in \operatorname{Sup}_{2}\left(G_{\text {right }}\right)$ and $\mathcal{A}=V(K, G)$. Then $\mathcal{A}$ is a CI-S-ring if and only if $K$ is $G$-transjugate. 
Proof. The statement of the lemma follows from [15, Theorem 2.6].

Let $K_{1}, K_{2} \in \operatorname{Sup}\left(G_{\text {right }}\right)$ such that $K_{1} \leq K_{2}$. Then $K_{1}$ is called a $G$-complete subgroup of $K_{2}$ if every $G$-regular subgroup of $K_{2}$ is $K_{2}$-conjugate to some $G$-regular subgroup of $K_{1}$ (see [15, Definition 2]). In this case we write $K_{1} \preceq_{G} K_{2}$. The relation $\preceq_{G}$ is a partial order on $\operatorname{Sup}\left(G_{\text {right }}\right)$. The set of the minimal elements of $\operatorname{Sup}_{2}\left(G_{\text {right }}\right)$ with respect to $\preceq_{G}$ is denoted by $\operatorname{Sup}_{2}^{\min }\left(G_{\text {right }}\right)$.

Lemma 4.2 ([20, Lemma 3.3]). Let $G$ be a finite group. If $V(K, G)$ is a CI-S-ring for every $K \in \operatorname{Sup}_{2}^{\min }\left(G_{\text {right }}\right)$ then $G$ is a DCI-group.

Remark 4.3. The condition that $V(K, G)$ is a CI-S-ring for every $K \in \operatorname{Sup}_{2}^{\min }\left(G_{\text {right }}\right)$ is equivalent to, say, that every schurian $S$-ring over $G$ is a CI- $S$-ring. The latter condition means that every 2-closed overgroup of $G_{\text {right }}$ is $G$-transjugate. However, 2-closed overgroup of $G_{\text {right }}$ may not be the automorphism group of a Cayley digraph over $G$. So the condition that the automorphism group of every Cayley digraph over $G$ is $G$-transjugate or, equivalently, that $G$ is a DCI-group, seems weaker than the condition of Lemma 4.2. It is a natural question whether there exists a DCI-group for which the condition of Lemma 4.2 does not hold.

We finish the section with the lemma that gives a sufficient condition for an $S$-ring to be a CI-S-ring. In order to formulate this condition, we need to introduce some further notations. Let $\mathcal{A}$ be a schurian $S$-ring over an abelian group $G$ and $L$ a normal $\mathcal{A}$-subgroup of $G$. Then the partition of $G$ into the $L$-cosets is $\operatorname{Aut}(\mathcal{A})$-invariant. The kernel of the action of $\operatorname{Aut}(\mathcal{A})$ on the latter cosets is denoted by $\operatorname{Aut}(\mathcal{A})_{G / L}$. Since $\operatorname{Aut}(\mathcal{A})_{G / L}$ is a normal subgroup of $\operatorname{Aut}(\mathcal{A})$, we can form the group $K=\operatorname{Aut}(\mathcal{A})_{G / L} G_{\text {right }}$. Clearly, $K \leq \operatorname{Aut}(\mathcal{A})$. From [15, Proposition 2.1] it follows that $K=K^{(2)}$.

Lemma 4.4. Let $\mathcal{A}$ be a schurian $S$-ring over an abelian group $G, L$ an $\mathcal{A}$-subgroup of $G$, and $K=\operatorname{Aut}(\mathcal{A})_{G / L} G_{\text {right }}$. Suppose that both $\mathcal{A}_{G / L}$ and $V(K, G)$ are CI-S-rings and $\mathcal{A}_{G / L}$ is normal. Then $\mathcal{A}$ is a CI-S-ring.

Proof. Firstly we prove that the $\operatorname{group} \operatorname{Aut}(\mathcal{A})^{G / L}$ is $G / L$-transjugate. Suppose that $F$ is a $G / L$-regular subgroup of $\operatorname{Aut}(\mathcal{A})^{G / L}$. The $S$-ring $\mathcal{A}_{G / L}$ is a CI-S-ring by the assumption of the lemma. So Lemma 4.1 implies that the group $\operatorname{Aut}\left(\mathcal{A}_{G / L}\right)$ is $G / L$-transjugate. Since $F \leq \operatorname{Aut}(\mathcal{A})^{G / L} \leq \operatorname{Aut}\left(\mathcal{A}_{G / L}\right)$, we conclude that $F$ and $(G / L)_{\text {right }}$ are $\operatorname{Aut}\left(\mathcal{A}_{G / L}\right)$ conjugate. However, $\mathcal{A}_{G / L}$ is normal and hence $F=(G / L)_{\text {right }}$. Therefore $\operatorname{Aut}(\mathcal{A})^{G / L}$ is $G / L$-transjugate.

Now let us show that $K \preceq_{G} \operatorname{Aut}(\mathcal{A})$. Let $H$ be a $G$-regular subgroup of $\operatorname{Aut}(\mathcal{A})$. Then $H^{G / L}$ is abelian transitive subgroup of $\operatorname{Aut}(\mathcal{A})^{G / L}$ and hence $H^{G / L}$ is regular on $G / L$. Therefore $H^{G / L} \cong(G / L)_{\text {right }}=\left(G_{\text {right }}\right)^{G / L}$. There exists $\gamma \in \operatorname{Aut}(\mathcal{A})$ such that $\left(H^{G / L}\right)^{\gamma^{G / L}}=(G / L)_{\text {right }}=\left(G_{\text {right }}\right)^{G / L}$ because $\operatorname{Aut}(\mathcal{A})^{G / L}$ is $G / L$-transjugate. This yields that $H^{\gamma} \leq K$. Thus, $K \preceq_{G} \operatorname{Aut}(\mathcal{A})$.

Finally, let us prove that $\operatorname{Aut}(\mathcal{A})$ is $G$-transjugate. Again, let $H$ be a $G$-regular subgroup of $\operatorname{Aut}(\mathcal{A})$. Since $K \preceq_{G} \operatorname{Aut}(\mathcal{A})$, there exists $\gamma \in \operatorname{Aut}(\mathcal{A})$ such that $H^{\gamma} \leq$ $K$. The $S$-ring $V(K, G)$ is a CI-S-ring by the assumption of the lemma. So $K$ is $G$ transjugate by Lemma 4.1. Therefore $H^{\gamma}$ and $G_{\text {right }}$ are $K$-conjugate and hence $H$ and $G_{\text {right }}$ are $\operatorname{Aut}(\mathcal{A})$-conjugate. Thus, $\operatorname{Aut}(\mathcal{A})$ is $G$-transjugate and $\mathcal{A}$ is a CI-S-ring by Lemma 4.1. 
It should be mentioned that the proof of Lemma 4.4 is similar to the proof of [20, Lemma 3.6].

\section{Generalized wreath and star products}

Let $\mathcal{A}$ be an $S$-ring over a group $G$ and $S=U / L$ an $\mathcal{A}$-section of $G$. An $S$-ring $\mathcal{A}$ is called the $S$-wreath product or the generalized wreath product of $\mathcal{A}_{U}$ and $\mathcal{A}_{G / L}$ if $L \unlhd G$ and $L \leq \operatorname{rad}(X)$ for each basic set $X$ outside $U$. In this case we write $\mathcal{A}=\mathcal{A}_{U} 2_{S} \mathcal{A}_{G / L}$ and omit $S$ when $U=L$. The construction of the generalized wreath product of $S$-rings was introduced in [12].

The $S$-wreath product is called nontrivial or proper if $L \neq\{e\}$ and $U \neq G$. An $S$-ring $\mathcal{A}$ is said to be decomposable if $\mathcal{A}$ is the nontrivial $S$-wreath product for some $\mathcal{A}$-section $S$ of $G$; otherwise $\mathcal{A}$ is said to be indecomposable. We say that an $\mathcal{A}$-subgroup $U<G$ has a gwr-complement with respect to $\mathcal{A}$ if there exists a nontrivial normal $\mathcal{A}$-subgroup $L$ of $G$ such that $L \leq U$ and $\mathcal{A}$ is the $S$-wreath product, where $S=U / L$.

Lemma 5.1 ([19, Theorem 1.1]). Let $G \in \mathcal{E}, \mathcal{A}$ an $S$-ring over $G$, and $S=U / L$ an $\mathcal{A}$ section of $G$. Suppose that $\mathcal{A}$ is the nontrivial $S$-wreath product and the $S$-rings $\mathcal{A}_{U}$ and $\mathcal{A}_{G / L}$ are CI-S-rings. Then $\mathcal{A}$ is a CI-S-ring whenever

$$
\operatorname{Aut}_{S}\left(\mathcal{A}_{S}\right)=\operatorname{Aut}_{U}\left(\mathcal{A}_{U}\right)^{S} \operatorname{Aut}_{G / L}\left(\mathcal{A}_{G / L}\right)^{S} .
$$

In particular, $\mathcal{A}$ is a $\mathrm{CI}-S$-ring if

$$
\operatorname{Aut}_{S}\left(\mathcal{A}_{S}\right)=\operatorname{Aut}_{U}\left(\mathcal{A}_{U}\right)^{S} \quad \text { or } \operatorname{Aut}_{S}\left(\mathcal{A}_{S}\right)=\operatorname{Aut}_{G / L}\left(\mathcal{A}_{G / L}\right)^{S} .
$$

Lemma 5.2 ([19, Proposition 4.1]). In the conditions of Lemma 5.1, suppose that $\mathcal{A}_{S}=$ $\mathbb{Z} S$. Then $\mathcal{A}$ is a CI-S-ring. In particular, if $U=L$ then $\mathcal{A}$ is a CI-S-ring.

Lemma 5.3 ([20, Lemma 4.2]). In the conditions of Lemma 5.1, suppose that at least one of the $S$-rings $\mathcal{A}_{U}$ and $\mathcal{A}_{G / L}$ is cyclotomic and $\mathcal{A}_{S}$ is Cayley minimal. Then $\mathcal{A}$ is a CI-S-ring.

Lemma 5.4. Let $\mathcal{A}$ be an $S$-ring over an abelian group $G$. Suppose that $\mathcal{A}$ is the nontrivial $S=U / L$-wreath product for some $\mathcal{A}$-section $S=U / L$ and $L_{1}$ is an $\mathcal{A}$-subgroup containing $L$. Then $\mathcal{B}=V(K, G)$, where $K=\operatorname{Aut}(\mathcal{A})_{G / L_{1}} G_{\text {right }}$, is also the $S$-wreath product.

Proof. Since $K \leq \operatorname{Aut}(\mathcal{A})$, from Equations (3.1) and (3.2) it follows that

$$
\mathcal{B}=V(K, G) \geq V(\operatorname{Aut}(\mathcal{A}), G) \geq \mathcal{A} .
$$

So $U$ and $L$ are also $\mathcal{B}$-subgroups.

Let $\mathcal{C}=\mathbb{Z} U \imath_{S} \mathbb{Z}(G / L)$. The $S$-rings $\mathcal{C}_{U}$ and $\mathcal{C}_{G / L}$ are schurian and $\mathcal{C}_{S}$ is 2 -minimal because $\mathcal{C}_{S}=\mathbb{Z} S$. So $\mathcal{C}$ is schurian by [26, Corollary 10.3]. This implies that

$$
\mathcal{e}=V(\operatorname{Aut}(\mathcal{C}), G) \text {. }
$$

Every element from $\operatorname{Aut}(\mathcal{C})_{e}$ fixes every basic set of $\mathcal{C}$ and hence it fixes every $L$ coset. Since $L_{1} \geq L$, every element from $\operatorname{Aut}(\mathcal{C})_{e}$ fixes every $L_{1}$-coset. We conclude that 
$\operatorname{Aut}(\mathcal{C})_{e} \leq \operatorname{Aut}(\mathcal{A})_{G / L_{1}}$ and hence $\operatorname{Aut}(\mathcal{C}) \leq K$. Now from Equations (3.1) and (5.1) it follows that

$$
\mathcal{C}=V(\operatorname{Aut}(\mathcal{C}), G) \geq V(K, G)=\mathcal{B}
$$

The group $U$ is a $\mathcal{B}$ - and a $\mathcal{C}$-subgroup. Due to Equation (5.2), every basic set of $\mathcal{B}$ which lies outside $U$ is a union of some basic sets of $\mathcal{C}$ which lie outside $U$. So $L \leq \operatorname{rad}(X)$ for every $X \in \mathcal{S}(\mathcal{B})$ outside $U$. Thus, $\mathcal{B}$ is the $S$-wreath product.

Lemma 5.5. In the conditions of Lemma 5.1, suppose that: (1) every $S$-ring over $U$ is a CI-S-ring; (2) $\mathcal{A}_{G / L}$ is 2-minimal or normal. Then $\mathcal{A}$ is a CI-S-ring.

Proof. Let $\mathcal{B}=V(K, G)$, where $K=\operatorname{Aut}(\mathcal{A})_{G / L} G_{\text {right }}$. From Lemma 5.4 it follows that $\mathcal{B}$ is the $S$-wreath product. Since $L_{1}=L$, the definition of $\mathcal{B}$ implies that $\mathcal{B}_{G / L}=\mathbb{Z}(G / L)$ and hence $\mathcal{B}_{S}=\mathbb{Z} S$. Clearly, $\mathcal{B}_{G / L}$ is a CI- $S$-ring. The $S$-ring $\mathcal{B}_{U}$ is a CI- $S$-ring by the assumption of the lemma. Therefore $\mathcal{B}$ is a CI-S-ring by Lemma 5.2. The $S$-ring $\mathcal{A}_{G / L}$ is a CI- $S$-ring by the assumption of the lemma. Thus, $\mathcal{A}$ is a CI-S-ring by [20, Lemma 3.6] whenever $\mathcal{A}_{G / L}$ is 2 -minimal and by Lemma 4.4 whenever $\mathcal{A}_{G / L}$ is normal.

Let $V$ and $W$ be $\mathcal{A}$-subgroups. The $S$-ring $\mathcal{A}$ is called the star product of $\mathcal{A}_{V}$ and $\mathcal{A}_{W}$ if the following conditions hold:

(1) $V \cap W \unlhd W$;

(2) each $T \in \mathcal{S}(\mathcal{A})$ with $T \subseteq(W \backslash V)$ is a union of some $V \cap W$-cosets;

(3) for each $T \in \mathcal{S}(\mathcal{A})$ with $T \subseteq G \backslash(V \cup W)$ there exist $R \in \mathcal{S}\left(\mathcal{A}_{V}\right)$ and $S \in \mathcal{S}\left(\mathcal{A}_{W}\right)$ such that $T=R S$.

In this case we write $\mathcal{A}=\mathcal{A}_{V} \star \mathcal{A}_{W}$. The construction of the star product of $S$-rings was introduced in [15]. The star product is called nontrivial if $V \neq\{e\}$ and $V \neq G$. If $V \cap W=\{e\}$ then the star product is the usual tensor product of $\mathcal{A}_{V}$ and $\mathcal{A}_{W}$ (see [11, p. 5]). In this case we write $\mathcal{A}=\mathcal{A}_{V} \otimes \mathcal{A}_{W}$. One can check that if $\mathcal{A}=\mathcal{A}_{V} \otimes \mathcal{A}_{W}$ then $\operatorname{Aut}(\mathcal{A})=\operatorname{Aut}\left(\mathcal{A}_{V}\right) \times \operatorname{Aut}\left(\mathcal{A}_{W}\right)$. If $V \cap W \neq\{e\}$ then $\mathcal{A}$ is the nontrivial $V /(V \cap W)$ wreath product. Indeed, let $T \in \mathcal{S}(\mathcal{A})$ such that $T \nsubseteq V$. If $T \subseteq W \backslash V$ then $V \cap W \leq$ $\operatorname{rad}(T)$ by Condition (2) of the definition. If $T \subseteq G \backslash(V \cup W)$ then $T=R S$ for some $R \in \mathcal{S}\left(\mathcal{A}_{V}\right)$ and some $S \in \mathcal{S}\left(\mathcal{A}_{W}\right)$ such that $S \subseteq W \backslash V$ by Condition (3) of the definition. Since $V \cap W \leq \operatorname{rad}(S)$, we obtain $V \cap W \leq \operatorname{rad}(T)$.

Lemma 5.6. Let $G \in \mathcal{E}$ and $\mathcal{A}$ a schurian $S$-ring over $G$. Suppose that $\mathcal{A}=\mathcal{A}_{V} \star \mathcal{A}_{W}$ for some $\mathcal{A}$-subgroups $V$ and $W$ of $G$ and the $S$-rings $\mathcal{A}_{V}$ and $\mathcal{A}_{W /(V \cap W)}$ are CI-S-rings. Then $\mathcal{A}$ is a CI-S-ring.

Proof. The statement of the lemma follows from [18, Proposition 3.2, Theorem 4.1].

Lemma 5.7 ([13, Lemma 2.8]). Let $\mathcal{A}$ be an $S$-ring over an abelian group $G=G_{1} \times G_{2}$. Assume that $G_{1}$ and $G_{2}$ are $\mathcal{A}$-groups. Then $\mathcal{A}=\mathcal{A}_{G_{1}} \otimes \mathcal{A}_{G_{2}}$ whenever $\mathcal{A}_{G_{1}}$ or $\mathcal{A}_{G_{2}}$ is the group ring.

Lemma 5.8. In the conditions of Lemma 5.1, suppose that $|G: U|$ is a prime and there exists $X \in \mathcal{S}\left(\mathcal{A}_{G / L}\right)$ outside $S$ with $|X|=1$. Then $\mathcal{A}$ is a CI-S-ring. 
Proof. Let $X=\{x\}$ for some $x \in G / L$. Due to $G \in \mathcal{E}$, we conclude that $|\langle x\rangle|$ is prime. So $|\langle x\rangle \cap S|=1$ because $x$ lies outside $S$. Since $|G: U|$ is a prime, $G / L=\langle x\rangle \times S$. Note that $\mathcal{A}_{\langle x\rangle}=\mathbb{Z}\langle x\rangle$. Therefore

$$
\mathcal{A}_{G / L}=\mathbb{Z}\langle x\rangle \otimes \mathcal{A}_{S}
$$

by Lemma 5.7 .

Let $\varphi \in \operatorname{Aut}_{S}\left(\mathcal{A}_{S}\right)$. Define $\psi \in \operatorname{Aut}(G / L)$ in the following way:

$$
\psi^{S}=\varphi, x^{\psi}=x
$$

Then $\psi \in \operatorname{Aut}_{G / L}\left(\mathcal{A}_{G / L}\right)$ because $\mathcal{A}_{G / L}=\mathbb{Z}\langle x\rangle \otimes \mathcal{A}_{S}$. We obtain that

$$
\operatorname{Aut}_{G / L}\left(\mathcal{A}_{G / L}\right)^{S} \geq \operatorname{Aut}_{S}\left(\mathcal{A}_{S}\right),
$$

and hence $\operatorname{Aut}_{G / L}\left(\mathcal{A}_{G / L}\right)^{S}=\operatorname{Aut}_{S}\left(\mathcal{A}_{S}\right)$. Thus, $\mathcal{A}$ is a CI-S-ring by Lemma 5.1 .

\section{$6 \quad p$-S-rings}

Let $p$ be a prime. An $S$-ring $\mathcal{A}$ over a $p$-group $G$ is called a $p$-S-ring if every basic set of $\mathcal{A}$ has a $p$-power size. Clearly, if $|G|=p$ then $\mathcal{A}=\mathbb{Z} G$. In the next three lemmas $G$ is a $p$-group and $\mathcal{A}$ is a $p$ - $S$-ring over $G$.

Lemma 6.1. If $\mathcal{B} \geq \mathcal{A}$ then $\mathcal{B}$ is a $p$-S-ring.

Proof. The statement of the lemma follows from [29, Theorem 1.1].

Lemma 6.2. Let $S=U / L$ be an $\mathcal{A}$-section of $G$. Then $\mathcal{A}_{S}$ is a $p$-S-ring.

Proof. From Lemma 2.1 it follows that for every $X \in \mathcal{S}(\mathcal{A})$ the number $\lambda=|X \cap L x|$ does not depend on $x \in X$. So $\lambda$ divides $|X|$ and hence $\lambda$ is a $p$-power. Let $\pi: G \rightarrow G / L$ be the canonical epimorphism. Note that $|\pi(X)|=|X| / \lambda$ and hence $|\pi(X)|$ is a $p$-power. Therefore every basic set of $\mathcal{A}_{S}$ has a $p$-power size. Thus, $\mathcal{A}_{S}$ is a $p$ - $S$-ring.

Lemma 6.3 ([13, Proposition 2.13]). The following statements hold:

(1) $\left|\mathbf{O}_{\theta}(\mathcal{A})\right|>1$;

(2) there exists a chain of $\mathcal{A}$-subgroups $\{e\}=G_{0}<G_{1}<\cdots<G_{s}=G$ such that $\left|G_{i+1}: G_{i}\right|=$ p for every $i \in\{0, \ldots, s-1\}$.

Lemma 6.4. Let $G$ be an abelian group, $K \in \operatorname{Sup}_{2}^{\min }\left(G_{\text {right }}\right)$, and $\mathcal{A}=V(K, G)$. Suppose that $H$ is an $\mathcal{A}$-subgroup of $G$ such that $G / H$ is a p-group for some prime $p$. Then $\mathcal{A}_{G / H}$ is a $p$-S-ring.

Proof. The statement of the lemma follows from [18, Lemma 5.2].

\section{$7 \quad S$-rings over an abelian group of non-powerful order}

A number $n$ is called powerful if $p^{2}$ divides $n$ for every prime divisor $p$ of $n$. From now throughout this section $G=H \times P$, where $H$ is an abelian group and $P \cong C_{p}$, where $p$ is a prime coprime to $|H|$. Clearly, $|G|$ is non-powerful. Let $\mathcal{A}$ be an $S$-ring over $G, H_{1}$ a maximal $\mathcal{A}$-subgroup contained in $H$, and $P_{1}$ the least $\mathcal{A}$-subgroup containing $P$. Note that $H_{1} P_{1}$ is an $\mathcal{A}$-subgroup. 
Lemma 7.1 ([20, Lemma 6.3]). In the above notations, if $H_{1} \neq\left(H_{1} P_{1}\right)_{p^{\prime}}$, the Hall $p^{\prime}$ subgroup of $H_{1} P_{1}$, then $\mathcal{A}_{H_{1} P_{1}}=\mathcal{A}_{H_{1}} \star \mathcal{A}_{P_{1}}$.

Lemma 7.2 ([27, Proposition 15]). In the above notations, if $\mathcal{A}_{H_{1} P_{1} / H_{1}} \cong \mathbb{Z} C_{p}$ then $\mathcal{A}_{H_{1} P_{1}}=\mathcal{A}_{H_{1}} \star \mathcal{A}_{P_{1}}$.

Lemma 7.3 ([11, Lemma 6.2]). In the above notations, suppose that $H_{1}<H$. Then one of the following statements holds:

(1) $\mathcal{A}=\mathcal{A}_{H_{1}} \prec \mathcal{A}_{G / H_{1}}$ with $\operatorname{rk}\left(\mathcal{A}_{G / H_{1}}\right)=2$;

(2) $\mathcal{A}=\mathcal{A}_{H_{1} P_{1}} 2_{S} \mathcal{A}_{G / P_{1}}$, where $S=H_{1} P_{1} / P_{1}$ and $P_{1}<G$.

\section{$8 \quad S$-rings over $C_{2}^{n}, n \leq 5$}

All $S$-rings over the groups $C_{2}^{n}$, where $n \leq 5$, were enumerated with the help of the GAP package COCO2P [16]. The list of all $S$-rings over these groups is available on the web-page [30] (see also [35]). The next lemma is an immediate consequence of the above computational results (see also [11, Theorem 1.2]).

Lemma 8.1. Every $S$-ring over $C_{2}^{n}$, where $n \leq 5$, is schurian.

To prove Theorem 1.1, we will show that every schurian $S$-ring over $C_{2}^{5} \times C_{p}$ is CI. Since the most of schurian $S$-rings over $C_{2}^{5} \times C_{p}$ are generalized wreath or star products of $S$-rings over its proper subgroups, we need to check that all schurian $S$-rings over proper subgroups of $C_{2}^{5} \times C_{p}$ are CI. In this section we will do it for $G \cong C_{2}^{n}$, where $n \leq 5$. Note that $G$ is a DCI-group by $[2,7]$ but this does not imply that every $S$-ring over $G$ is CI (see Remark 4.3). We will describe $2-S$-rings over $G$ using computational results and check that all $S$-rings over $G$ are CI. Until the end of the section $G$ is an elementary abelian 2-group of rank $n$ and $\mathcal{A}$ is a 2 - $S$-ring over $G$.

Lemma 8.2. Let $n \leq 3$. Then $\mathcal{A}$ is cyclotomic. Moreover, $\mathcal{A}$ is Cayley minimal except for the case when $n=3$ and $\mathcal{A} \cong \mathbb{Z} C_{2} \prec \mathbb{Z} C_{2} \prec \mathbb{Z} C_{2}$.

Proof. The first part of the lemma follows from [20, Lemma 5.2]; the second part follows from [20, Lemma 5.3].

Analyzing the lists of all $S$-rings over $C_{2}^{4}$ and $C_{2}^{5}$ available on the web-page [30], we conclude that up to isomorphism there are exactly nineteen 2 - $S$-rings over $G$ if $n=4$ and there are exactly one hundred $2-S$-rings over $G$ if $n=5$. It can can be established by inspecting the above 2 - $S$-rings one after the other that there are exactly fifteen decomposable and four indecomposable 2 - $S$-rings over $G$ if $n=4$ and there are exactly ninety six decomposable and four indecomposable 2-S-rings over $G$ if $n=5$.

Lemma 8.3. Let $n \in\{4,5\}$ and $\mathcal{A}$ indecomposable. Then $\mathcal{A}$ is normal. If in addition $n=5$ then $\mathcal{A} \cong \mathbb{Z} C_{2} \otimes \mathcal{A}^{\prime}$, where $\mathcal{A}^{\prime}$ is indecomposable 2 -S-ring over $C_{2}^{4}$.

Proof. Let $n=4$. One can compute $|\operatorname{Aut}(\mathcal{A})|$ and $\left|N_{\text {Aut }(\mathcal{A})}\left(G_{\text {right }}\right)\right|$ using the GAP package COCO2P [16]. It turns out that for each of the four indecomposable 2 - $S$-rings over $G$ the equality

$$
|\operatorname{Aut}(\mathcal{A})|=\left|N_{\operatorname{Aut}(\mathcal{A})}\left(G_{\text {right }}\right)\right|
$$


is attained. So every indecomposable 2 - $S$-ring over $G$ is normal whenever $n=4$.

Let $n=5$. The straightforward check for each of the four indecomposable 2-S-rings over $G$ yields that $\mathcal{A}=\mathcal{A}_{H} \otimes \mathbb{Z} L$, where $H \cong C_{2}^{4}, L \cong C_{2}$, and $\mathcal{A}_{H}$ is indecomposable 2 - $S$-ring. Clearly, $\mathbb{Z} L$ is normal. By the above paragraph, $\mathcal{A}_{H}$ is normal. Since $\operatorname{Aut}(\mathcal{A})=$ $\operatorname{Aut}\left(\mathcal{A}_{H}\right) \times \operatorname{Aut}\left(\mathcal{A}_{L}\right)$, we obtain that $\mathcal{A}$ is normal.

Note that if $p>2$ then Lemma 8.3 does not hold. In fact, if $p>2$ then there exists an indecomposable $p$ - $S$-ring over $C_{p}^{5}$ which is not normal (see [13, Lemma 6.4]).

Lemma 8.4. Let $n \leq 5$. Then $\mathcal{A}$ is normal whenever one of the following statements holds:

(1) $\mathcal{A}$ is indecomposable;

(2) $\left|G: \mathbf{O}_{\theta}(\mathcal{A})\right|=2$;

(3) $n=4$ and $\mathcal{A} \cong\left(\mathbb{Z} C_{2} \succ \mathbb{Z} C_{2}\right) \otimes\left(\mathbb{Z} C_{2} \succ \mathbb{Z} C_{2}\right)$.

Proof. If $n \leq 3$ and $\mathcal{A}$ is indecomposable then $\mathcal{A}=\mathbb{Z} G$ by [20, Lemma 5.2]. Clearly, in this case $\mathcal{A}$ is normal. If $n \in\{4,5\}$ and $\mathcal{A}$ is indecomposable then $\mathcal{A}$ is normal by Lemma 8.3. There are exactly $n-12$-S-rings over $G$ for which Statement (2) of the lemma holds. For every $\mathcal{A}$ isomorphic to one of these 2 -S-rings and for $\mathcal{A} \cong\left(\mathbb{Z} C_{2}\left\langle\mathbb{Z} C_{2}\right) \otimes\right.$ $\left(\mathbb{Z} C_{2} \succ \mathbb{Z} C_{2}\right)$ one can compute $|\operatorname{Aut}(\mathcal{A})|$ and $\left|N_{\text {Aut }(\mathcal{A})}\left(G_{\text {right }}\right)\right|$ using the GAP package COCO2P [16]. It turns out that in each case the equality $|\operatorname{Aut}(\mathcal{A})|=\left|N_{\text {Aut }(\mathcal{A})}\left(G_{\text {right }}\right)\right|$ holds and hence $\mathcal{A}$ is normal.

Lemma 8.5. Let $n=4$. Then $\mathcal{A}$ is cyclotomic.

Proof. If $\mathcal{A}$ is decomposable then $\mathcal{A}$ is cyclotomic by [20, Lemma 5.6]. If $\mathcal{A}$ is indecomposable then $\mathcal{A}$ is normal by Lemma 8.3. This implies that

$$
\operatorname{Aut}(\mathcal{A})_{e}=\left(N_{\operatorname{Aut}(\mathcal{A})}\left(G_{\text {right }}\right)\right)_{e} \leq \operatorname{Aut}(G)
$$

The $S$-ring $\mathcal{A}$ is schurian by Lemma 8.1. So from Equation (3.2) it follows that $\mathcal{A}=$ $V(\operatorname{Aut}(\mathcal{A}), G)$ and hence $\mathcal{A}=\operatorname{Cyc}\left(\operatorname{Aut}(\mathcal{A})_{e}, G\right)$.

Lemma 8.6. Let $n=5$. Suppose that $\mathcal{A}$ is decomposable and $\left|\mathbf{O}_{\theta}(\mathcal{A})\right|=8$. Then $\mathcal{A}$ is cyclotomic.

Proof. Let $\mathcal{A}$ be the nontrivial $S$-wreath product for some $\mathcal{A}$-section $S=U / L$. Note that $|U| \leq 16,|G / L| \leq 16$, and $|S| \leq 8$. The $S$-rings $\mathcal{A}_{U}, \mathcal{A}_{G / L}$, and $\mathcal{A}_{S}$ are 2 - $S$-rings by Lemma 6.2. So each of these $S$-rings is cyclotomic by Lemma 8.2 whenever the order of the corresponding group is at most 8 and by Lemma 8.5 otherwise. Since $\left|\mathbf{O}_{\theta}(\mathcal{A})\right|=8$, we conclude that $|S| \leq 4$ or $|S|=8$ and $\left|\mathbf{O}_{\theta}\left(\mathcal{A}_{S}\right)\right| \geq 4$. In both cases $\mathcal{A}_{S}$ is Cayley minimal by Lemma 8.2. This implies that

$$
\operatorname{Aut}_{U}\left(\mathcal{A}_{U}\right)^{S}=\operatorname{Aut}_{G / L}\left(\mathcal{A}_{G / L}\right)^{S}=\operatorname{Aut}_{S}\left(\mathcal{A}_{S}\right) .
$$

Now from [20, Lemma 4.3] it follows that $\mathcal{A}$ is cyclotomic. 
In the next two lemmas we establish some properties of decomposable 2 - $S$-rings over $G \cong C_{2}^{5}$ whose thin radical is of size 2 or 4 . These properties will be used in the proof of Theorem 1.1. The statements of Lemma 8.7 and Lemma 8.8 can be verified by analysis of computational results obtained with the help of the GAP package COCO2P [16]. For every decomposable 2 - $S$-ring $\mathcal{A}$ with $\left|\mathbf{O}_{\theta}(\mathcal{A})\right| \in\{2,4\}$ over $G$ (see the list [30]), we compute all $\mathcal{A}$-subgroups, automorphism groups, and Cayley automorphism groups of some restrictions and quotients.

Lemma 8.7. Let $n=5$. Suppose that $\mathcal{A}$ is decomposable and $\left|\mathbf{O}_{\theta}(\mathcal{A})\right|=4$. Then one of the following statements holds:

(1) there exists an $\mathcal{A}$-subgroup $L \leq \mathbf{O}_{\theta}(\mathcal{A})$ of order 2 such that $\mathcal{A}=\mathbb{Z} \mathbf{O}_{\theta}(\mathcal{A}){ }_{l_{S}} \mathcal{A}_{G / L}$, where $S=\mathbf{O}_{\theta}(\mathcal{A}) / L$;

(2) $\left|\operatorname{Aut}_{G}(\mathcal{A})\right| \geq\left|\operatorname{Aut}_{U}\left(\mathcal{A}_{U}\right)\right|$ for every $\mathcal{A}$-subgroup $U$ with $|U|=16$ and $U \geq$ $\mathbf{O}_{\theta}(\mathcal{A})$

(3) $\mathcal{A}$ is normal;

(4) there exist an $\mathcal{A}$-subgroup $L \leq \mathbf{O}_{\theta}(\mathcal{A})$ and $X \in \mathcal{S}(\mathcal{A})$ such that $|L|=|X|=2$, $L \neq \operatorname{rad}(X)$, and $\mathcal{A}_{G / L}$ is normal.

Lemma 8.8. Let $n=5$. Suppose that $\mathcal{A}$ is decomposable, $\left|\mathbf{O}_{\theta}(\mathcal{A})\right|=2$, and there exists $X \in \mathcal{S}(\mathcal{A})$ with $|X|>1$ and $|\operatorname{rad}(X)|=1$. Then $|X|=4$ and one of the following statements holds:

(1) $\mathcal{A} \cong \mathcal{B} \backslash \mathbb{Z} C_{2}$, where $\mathcal{B}$ is a 2 -S-ring over $C_{2}^{4}$;

(2) $\left|\operatorname{Aut}_{G}(\mathcal{A})\right| \geq\left|\operatorname{Aut}_{U}\left(\mathcal{A}_{U}\right)\right|$ for every $\mathcal{A}$-subgroup $U$ with $|U|=16$;

(3) there exists an $\mathcal{A}$-subgroup $L$ such that $|L| \in\{2,4\}$ and $\mathcal{A}_{G / L}$ is normal.

Lemma 8.9. Let $D \in \mathcal{E}$ such that every $S$-ring over a proper section of $D$ is $\mathrm{CI}, \mathcal{D}$ an $S$-ring over $D$, and $S=U / L$ a $\mathcal{D}$-section. Suppose that $\mathcal{D}$ is the nontrivial $S$-wreath product. Then $\mathcal{D}$ is a CI-S-ring whenever $D / L \cong C_{2}^{k}$ for some $k \leq 4$ and $\mathcal{D}_{D / L}$ is a 2-S-ring.

Proof. The $S$-ring $\mathcal{D}_{D / L}$ is cyclotomic by Lemma 8.2 whenever $|D / L| \leq 8$ and by Lemma 8.5 whenever $|D / L|=16$. The $S$-ring $\mathcal{D}_{S}$ is a 2 - $S$-ring by Lemma 6.2. If $\mathcal{D}_{S} \nsucceq \mathbb{Z} C_{2} \prec \mathbb{Z} C_{2} \prec \mathbb{Z} C_{2}$ then $\mathcal{D}_{S}$ is Cayley minimal by Lemma 8.2. The $S$-rings $\mathcal{D}_{U}$ and $\mathcal{D}_{D / L}$ are CI-S-rings by the assumption of the lemma. So $\mathcal{D}$ is a CI-S-ring by Lemma 5.3.

Assume that

$$
\mathcal{D}_{S} \cong \mathbb{Z} C_{2} \prec \mathbb{Z} C_{2} \succ \mathbb{Z} C_{2} \text {. }
$$

In this case $|D / L|=16,|S|=8$, and there exists the least $\mathcal{D}_{S}$-subgroup $A$ of $S$ of order 2 . Every basic set of $\mathcal{D}_{D / L}$ outside $S$ is contained in an $S$-coset because $\mathcal{D}_{(D / L) / S} \cong \mathbb{Z} C_{2}$. So $\operatorname{rad}(X)$ is a $\mathcal{D}_{S}$-subgroup for every $X \in \mathcal{S}\left(\mathcal{D}_{D / L}\right)$ outside $S$. If $|\operatorname{rad}(X)|>1$ for every $X \in \mathcal{S}\left(\mathcal{D}_{D / L}\right)$ outside $S$ then $\mathcal{D}_{D / L}$ is the $S / A$-wreath product because $A$ is the least $\mathcal{D}_{S}$-subgroup. This implies that $\mathcal{D}$ is the $U / \pi^{-1}(A)$-wreath product, where $\pi: D \rightarrow D / L$ is the canonical epimorphism. One can see that $\left|D / \pi^{-1}(A)\right| \leq 8$ and $\left|U / \pi^{-1}(A)\right| \leq$ 4. The $S$-rings $\mathcal{D}_{D / \pi^{-1}(A)}$ and $\mathcal{D}_{U / \pi^{-1}(A)}$ are 2 - $S$-rings by Lemma 6.2. The $S$-ring $\mathcal{D}_{D / \pi^{-1}(A)}$ is cyclotomic by Lemma 8.2 and the $S$-ring $\mathcal{D}_{U / \pi^{-1}(A)}$ is Cayley minimal by 
Lemma 8.2. The $S$-rings $\mathcal{D}_{U}$ and $\mathcal{D}_{D / \pi^{-1}(A)}$ are CI-S-rings by the assumption of the lemma. Thus, $\mathcal{D}$ is a CI- $S$-ring by Lemma 5.3.

Suppose that there exists a basic set $X$ of $\mathcal{D}_{D / L}$ outside $S$ with $|\operatorname{rad}(X)|=1$. If $\mathcal{D}_{D / L}$ is decomposable then

$$
\operatorname{Aut}_{D / L}\left(\mathcal{D}_{D / L}\right)^{S}=\operatorname{Aut}_{S}\left(\mathcal{D}_{S}\right)
$$

by [20, Lemma 5.8]. Therefore $\mathcal{D}$ is a CI- $S$-ring by Lemma 5.1 .

If $\mathcal{D}_{D / L}$ is indecomposable then $\mathcal{D}_{D / L}$ is normal by Lemma 8.3. So all conditions of Lemma 5.5 hold for $\mathcal{D}$. Thus, $\mathcal{D}$ is a $\mathrm{CI}-S$-ring.

Lemma 8.10. Let $n \leq 5$. Then every $S$-ring over $G$ is a CI-S-ring.

Proof. Every $S$-ring over $G$ is schurian by Lemma 8.1. So to prove the lemma, it is sufficient to prove that $\mathcal{B}=V(K, G)$ is a CI-S-ring for every $K \in \operatorname{Sup}_{2}^{\min }\left(G_{\text {right }}\right)$ (see Remark 4.3). The $S$-ring $\mathcal{B}$ is a 2 - $S$-ring by Lemma 6.4. If $n \leq 4$ then $\mathcal{B}$ is CI by [20, Lemma 5.7]. Thus, if $n=4$ then the statement of the lemma holds.

Let $n=5$. Suppose that $\mathcal{B}$ is indecomposable. Then the second part of Lemma 8.3 implies $\mathcal{B} \cong \mathbb{Z} C_{2} \otimes \mathcal{B}^{\prime}$, where $\mathcal{B}^{\prime}$ is indecomposable 2 -S-ring over $C_{2}^{4}$. Since $\mathcal{B}$ is schurian by Lemma 8.1 and every $S$-ring over an elementary abelian group of rank at most 4 is CI by the above paragraph, we conclude that $\mathcal{B}$ is a CI- $S$-ring by Lemma 5.6.

Now suppose that $\mathcal{B}$ is decomposable, i.e. $\mathcal{B}$ is the nontrivial $S=U / L$-wreath product for some $\mathcal{B}$-section $S=U / L$. Clearly, $|G / L| \leq 16$. The $S$-ring $\mathcal{B}_{G / L}$ is a 2 - $S$-ring by Lemma 6.2. Since every $S$-ring over an elementary abelian group of rank at most 4 is CI, $\mathcal{B}$ is a CI- $S$-ring by Lemma 8.9.

\section{Proof of Theorem 1.1}

Let $G=H \times P$, where $H \cong C_{2}^{5}$ and $P \cong C_{p}$, where $p$ is a prime. These notations are valid until the end of the paper. If $p=2$ then $G$ is not a DCI-group by [28]. So in view of Lemma 4.2, to prove Theorem 1.1, it is sufficient to prove the following theorem.

Theorem 9.1. Let $p$ be an odd prime and $K \in \operatorname{Sup}_{2}^{\min }\left(G_{\text {right }}\right)$. Then $\mathcal{A}=V(K, G)$ is a CI-S-ring.

The proof of Proposition 9.1 will be given at the end of the section. We start with the next lemma concerned with proper sections of $G$.

Lemma 9.2. Let $S$ be a section of $G$ such that $S \neq G$. Then every schurian $S$-ring over $S$ is $a$ CI-S-ring.

Proof. If $S \cong C_{2}^{n}$ for some $n \leq 5$ then we are done by Lemma 8.10. Suppose that $S \cong$ $C_{2}^{n} \times C_{p}$ for some $n \leq 4$. Then the statement of the lemma follows from [20, Remark 3.4] whenever $n \leq 3$ and from [20, Remark 3.4, Theorem 7.1] whenever $n=4$.

A key step towards the proof of Theorem 9.1 is the following lemma.

Lemma 9.3. Let $\mathcal{A}$ be an $S$-ring over $G$ and $U$ an $\mathcal{A}$-subgroup with $U \geq P$. Suppose that $P$ is an $\mathcal{A}$-subgroup, $\mathcal{A}$ is the nontrivial $S$-wreath product, where $S=U / P,|S|=16$, and $\mathcal{A}_{G / P}$ is a 2 -S-ring. Then $\mathcal{A}$ is a CI-S-ring. 
Proof. Firstly we prove two lemmas concerned with some special cases of Lemma 9.3.

Lemma 9.4. Suppose that $S$ has a gwr-complement with respect to $\mathcal{A}_{G / P}$. Then $\mathcal{A}$ is a CI-S-ring.

Proof. The condition of the lemma implies that there exists an $\mathcal{A}_{G / P}$-subgroup $A$ such that $\mathcal{A}_{G / P}$ is the nontrivial $S / A$-wreath product. This means that $\mathcal{A}$ is the nontrivial $U / \pi^{-1}(A)$-wreath product, where $\pi: G \rightarrow G / P$ is the canonical epimorphism. Note that $\left|G / \pi^{-1}(A)\right| \leq 16$ and $\mathcal{A}_{G / \pi^{-1}(A)} \cong \mathcal{A}_{(G / P) / A}$ is a 2 -S-ring by Lemma 6.2. Therefore $\mathcal{A}$ is a CI-S-ring by Lemma 9.2 and Lemma 8.9 .

Lemma 9.5. Suppose that $S$ does not have a gwr-complement with respect to $\mathcal{A}_{G / P}$. Then

$$
\left|\operatorname{Aut}_{G / P}\left(\mathcal{A}_{G / P}\right)^{S}\right|=\left|\operatorname{Aut}_{G / P}\left(\mathcal{A}_{G / P}\right)\right| .
$$

Proof. To prove the lemma it is sufficient to prove that the group

$$
\left(\operatorname{Aut}_{G / P}\left(\mathcal{A}_{G / P}\right)\right)_{S}=\left\{\varphi \in \operatorname{Aut}_{G / P}\left(\mathcal{A}_{G / P}\right): \varphi^{S}=\operatorname{id}_{S}\right\}
$$

is trivial. Let $\varphi \in\left(\operatorname{Aut}_{G / P}\left(\mathcal{A}_{G / P}\right)\right)_{S}$. Put $\mathcal{C}=\operatorname{Cyc}(\langle\varphi\rangle, G / P)$. Clearly, $\langle\varphi\rangle \leq$ $\operatorname{Aut}\left(\mathcal{A}_{G / P}\right)$. So from Equations (3.1) and (3.2) it follows that $\mathcal{C} \geq \mathcal{A}_{G / P}$. Lemma 6.1 yields that $\mathcal{C}$ is a $2-S$-ring. Since $\varphi^{S}=\mathrm{id}_{S}$, we conclude that $\mathbf{O}_{\theta}(\mathcal{C}) \geq S$.

If $\mathcal{C} \neq \mathbb{Z}(G / P)$ then $\mathbf{O}_{\theta}(\mathcal{C})=S$. Therefore $\mathcal{C}=\mathbb{Z} S \imath_{S / A} \mathbb{Z}((G / P) / A)$ for some $\mathcal{C}$ subgroup $A$ by Statement (i) of [19, Proposition 4.3]. This implies that $\mathcal{A}_{G / P}=\mathcal{A}_{S}{ }_{S / A}$ $\mathcal{A}_{((G / P) / A}$ because $\mathcal{C} \geq \mathcal{A}_{G / P}$ and $S$ is both $\mathcal{A}_{G / P}$, $\mathcal{C}$-subgroup. We obtain a contradiction with the assumption of the lemma. Thus, $\mathcal{C}=\mathbb{Z}(G / P)$ and hence $\varphi$ is trivial. So the group $\left(\operatorname{Aut}_{G / P}\left(\mathcal{A}_{G / P}\right)\right)_{S}$ is trivial.

If $\mathcal{A}_{G / P}$ is indecomposable then $\mathcal{A}_{G / P}$ is normal by Lemma 8.3. So $\mathcal{A}$ is a CI- $S$-ring by Lemma 9.2 and Lemma 5.5. Further we assume that $\mathcal{A}_{G / P}$ is decomposable. Due to Lemma 9.4, we may assume also that

$$
S \text { does not have a gwr-complement with respect to } \mathcal{A}_{G / P} \text {. }
$$

If there exists $X \in \mathcal{S}\left(\mathcal{A}_{G / P}\right)$ outside $S$ with $|X|=1$ then $\mathcal{A}$ is a CI-S-ring by Lemma 9.2 and Lemma 5.8. So we may assume that

$$
\mathbf{O}_{\theta}\left(\mathcal{A}_{G / P}\right) \leq S
$$

Note that $\left|\mathbf{O}_{\theta}\left(\mathcal{A}_{G / P}\right)\right|>1$ by Statement (1) of Lemma 6.3 and $\left|\mathbf{O}_{\theta}\left(\mathcal{A}_{G / P}\right)\right| \leq 16$ by Equation (9.2). So $\left|\mathbf{O}_{\theta}\left(\mathcal{A}_{G / P}\right)\right| \in\{2,4,8,16\}$. We divide the rest of the proof into four cases depending on $\left|\mathbf{O}_{\theta}\left(\mathcal{A}_{G / P}\right)\right|$.

Case 1: $\left|\mathbf{O}_{\theta}\left(\mathcal{A}_{G / P}\right)\right|=16$.

Due to Equation (9.2), we conclude that $\mathcal{A}_{S}=\mathbb{Z} S$. So $\mathcal{A}$ is a CI-S-ring by Lemma 9.2 and Lemma 5.2.

Case 2: $\left|\mathbf{O}_{\theta}\left(\mathcal{A}_{G / P}\right)\right|=8$.

Since $\mathcal{A}_{G / P}$ is decomposable, Lemma 8.6 implies that $\mathcal{A}_{G / P}$ is cyclotomic. The $S$-ring $\mathcal{A}_{S}$ is a $2-S$-ring by Lemma 6.2. In view of Equation (9.2), we obtain that $\left|\mathbf{O}_{\theta}\left(\mathcal{A}_{S}\right)\right|=8$. So Statement (ii) of [19, Proposition 4.3] yields that the $S$-ring $\mathcal{A}_{S}$ is Cayley minimal. Thus, $\mathcal{A}$ is a CI- $S$-ring by Lemma 9.2 and Lemma 5.3. 
Case 3: $\left|\mathbf{O}_{\theta}\left(\mathcal{A}_{G / P}\right)\right|=4$.

In this case one of the statements of Lemma 8.7 holds for $\mathcal{A}_{G / P}$. If Statement (1) of Lemma 8.7 holds for $\mathcal{A}_{G / P}$ then we obtain a contradiction with Equation (9.1).

If Statement (2) of Lemma 8.7 holds for $\mathcal{A}_{G / P}$ then $\left|\operatorname{Aut}_{G / P}\left(\mathcal{A}_{G / P}\right)\right| \geq\left|\operatorname{Aut}_{S}\left(\mathcal{A}_{S}\right)\right|$. From Lemma 9.5 it follows that $\left|\operatorname{Aut}_{G / P}\left(\mathcal{A}_{G / P}\right)^{S}\right|=\left|\operatorname{Aut}_{G / P}\left(\mathcal{A}_{G / P}\right)\right|$ and hence

$$
\left|\operatorname{Aut}_{G / P}\left(\mathcal{A}_{G / P}\right)^{S}\right| \geq\left|\operatorname{Aut}_{S}\left(\mathcal{A}_{S}\right)\right| .
$$

Since $\operatorname{Aut}_{G / P}\left(\mathcal{A}_{G / P}\right)^{S} \leq \operatorname{Aut}_{S}\left(\mathcal{A}_{S}\right)$, we conclude that $\operatorname{Aut}_{G / P}\left(\mathcal{A}_{G / P}\right)^{S}=\operatorname{Aut}_{S}\left(\mathcal{A}_{S}\right)$. Thus, $\mathcal{A}$ is a CI-S-ring by Lemma 9.2 and Lemma 5.1 .

If Statement (3) of Lemma 8.7 holds for $\mathcal{A}_{G / P}$ then $\mathcal{A}_{G / P}$ is normal. In this case $\mathcal{A}$ is a CI-S-ring by Lemma 9.2 and Lemma 5.5 .

Suppose that Statement (4) of Lemma 8.7 holds for $\mathcal{A}_{G / P}$, i.e. there exists an $\mathcal{A}_{G / P^{-}}$ subgroup $A \leq \mathbf{O}_{\theta}\left(\mathcal{A}_{G / P}\right)$ of order 2 and $X=\left\{x_{1}, x_{2}\right\} \in \mathcal{S}\left(\mathcal{A}_{G / P}\right)$ such that $\mathcal{A}_{(G / P) / A}$ is normal and $A \neq \operatorname{rad}(X)$. Let $L=\pi^{-1}(A)$, where $\pi: G \rightarrow G / P$ is the canonical epimorphism, and $\mathcal{B}=V(N, G)$, where $N=\operatorname{Aut}(\mathcal{A})_{G / L} G_{\text {right }}$.

Prove that $\mathcal{B}$ is a CI-S-ring. Lemma 5.4 implies that $\mathcal{B}$ is the $S$-wreath product. From Equations (3.1) and (3.2) it follows that $\mathcal{B} \geq \mathcal{A}$. So $\mathcal{B}_{G / P} \geq \mathcal{A}_{G / P}$ and hence $\mathcal{B}_{G / P}$ is a $2-S$-ring by Lemma 6.1. We obtain that $\mathcal{B}$ and $U$ satisfy the conditions of Lemma 9.3.

One can see that $X$ is a $\mathcal{B}_{G / P^{-}}$-set and

$$
\mathbf{O}_{\theta}\left(\mathcal{B}_{G / P}\right) \geq \mathbf{O}_{\theta}\left(\mathcal{A}_{G / P}\right)
$$

because $\mathcal{B}_{G / P} \geq \mathcal{A}_{G / P}$. The definition of $\mathcal{B}$ yields that every basic set of $\mathcal{B}$ is contained in an $L$-coset and hence every basic set of $\mathcal{B}_{G / P}$ is contained in an $A$-coset. Therefore

$$
\left\{x_{1}\right\},\left\{x_{2}\right\} \in \mathcal{S}\left(\mathcal{B}_{G / P}\right)
$$

because $X$ is a $\mathcal{B}_{G / P^{-}}$-set and $A \neq \operatorname{rad}(X)$. Now from Equations (9.3) and (9.4) it follows that

$$
\left|\mathbf{O}_{\theta}\left(\mathcal{B}_{G / P}\right)\right| \geq 8 \text {. }
$$

If $\mathcal{B}_{G / P}$ is indecomposable then $\mathcal{B}_{G / P}$ is normal by Lemma 8.3 and hence $\mathcal{B}$ is CI by Lemma 9.2 and Lemma 5.5. If $S$ has a gwr-complement with respect to $\mathcal{B}_{G / P}$ then $\mathcal{B}$ is CI by Lemma 9.4. If $\mathbf{O}_{\theta}\left(\mathcal{B}_{G / P}\right) \not S$ then $\mathcal{B}$ is CI by Lemma 9.2 and Lemma 5.8. Suppose that none of the above conditions does not hold for $\mathcal{B}$. Then, in view of Equation (9.5), $\mathcal{B}$ satisfies all conditions from one of the Cases 1 or 2 . Therefore, $\mathcal{B}$ is CI.

Clearly, $\mathcal{A}_{G / L} \cong \mathcal{A}_{(G / P) / A}$ and hence $\mathcal{A}_{G / L}$ is normal. Also $\mathcal{A}_{G / L}$ is CI by Lemma 9.2. The $S$-ring $\mathcal{B}$ is CI by the above paragraph. Thus, $\mathcal{A}$ is CI by Lemma 4.4.

Case 4: $\left|\mathbf{O}_{\theta}\left(\mathcal{A}_{G / P}\right)\right|=2$.

Let $A=\mathbf{O}_{\theta}\left(\mathcal{A}_{G / P}\right)$. Clearly, $A$ is the least $\mathcal{A}_{G / P}$-subgroup. If $|\operatorname{rad}(X)|>1$ for every $X \in \mathcal{S}\left(\mathcal{A}_{G / P}\right)$ outside $S$ then $A \leq \operatorname{rad}(X)$ for every $X \in \mathcal{S}\left(\mathcal{A}_{G / P}\right)$ outside $S$ and we obtain a contradiction with Equation (9.1). So there exists $X \in \mathcal{S}\left(\mathcal{A}_{G / P}\right)$ outside $S$ with $|\operatorname{rad}(X)|=1$. From Equation (9.2) it follows that $|X|>1$. Lemma 8.8 implies that $|X|=4$. The number $\lambda=|X \cap A x|$ does not depend on $x \in X$ by Lemma 2.1. If $\lambda=2$ then $A \leq \operatorname{rad}(X)$, a contradiction. Therefore

$$
\lambda=1 \text {. }
$$


One of the statements of Lemma 8.8 holds for $\mathcal{A}_{G / P}$. If Statement (1) of Lemma 8.8 holds for $\mathcal{A}_{G / P}$ then there exists $Y \in \mathcal{S}\left(\mathcal{A}_{G / P}\right)$ with $|Y|=16$ and $|\operatorname{rad}(Y)|=16$. Since $|S|=16$, we conclude that $Y$ lies outside $S$ and hence $Y=(G / P) \backslash S$. This means that $S$ is a gwr-complement to $S$ with respect to $\mathcal{A}_{G / P}$. However, this contradicts Equation (9.1).

If Statement (2) of Lemma 8.8 holds for $\mathcal{A}_{G / P}$ then $\left|\operatorname{Aut}_{G / P}\left(\mathcal{A}_{G / P}\right)\right| \geq\left|\operatorname{Aut}_{S}\left(\mathcal{A}_{S}\right)\right|$. So Lemma 9.5 implies that $\operatorname{Aut}_{G / P}\left(\mathcal{A}_{G / P}\right)^{S}=\operatorname{Aut}_{S}\left(\mathcal{A}_{S}\right)$. Therefore, $\mathcal{A}$ is $\mathrm{CI}$ by Lemma 9.2 and Lemma 5.1

Suppose that Statement (3) of Lemma 8.8 holds for $\mathcal{A}_{G / P}$, i.e. there exists an

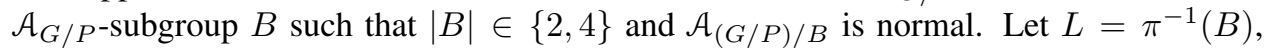
where $\pi: G \rightarrow G / P$ is the canonical epimorphism, and $\mathcal{B}=V(N, G)$, where $N=$ $\operatorname{Aut}(\mathcal{A})_{G / L} G_{\text {right }}$.

We prove that $\mathcal{B}$ is a CI-S-ring. As in Case 3, $\mathcal{B}$ is the $S$-wreath product by Lemma 5.4 and $\mathcal{B} \geq \mathcal{A}$ by Equations (3.1) and (3.2). So $\mathcal{B}_{G / P} \geq \mathcal{A}_{G / P}$ and hence $\mathcal{B}_{G / P}$ is a $2-S$-ring by Lemma 6.1. Therefore $\mathcal{B}$ and $U$ satisfy the conditions of Lemma 9.3.

Note that $X$ is a $\mathcal{B}_{G / P}$-set and Equation (9.3) holds because $\mathcal{B}_{G / P} \geq \mathcal{A}_{G / P}$. By the definition of $\mathcal{B}$, every basic set of $\mathcal{B}$ is contained in an $L$-coset and hence every basic set of $\mathcal{B}_{G / P}$ is contained in a $B$-coset. The set $X$ is a $\mathcal{B}_{G / P}$-set with $|X|=4$ and $|\operatorname{rad}(X)|=1$. So there exists $X_{1} \in \mathcal{S}\left(\mathcal{B}_{G / P}\right)$ such that

$$
X_{1} \subset X \text { and }\left|X_{1}\right| \in\{1,2\} .
$$

If $\left|X_{1}\right|=1$ then $X_{1} \subseteq \mathbf{O}_{\theta}\left(\mathcal{B}_{G / P}\right)$. If $\left|X_{1}\right|=2$ then $X_{1}$ is a coset by a $\mathcal{B}_{G / P^{- \text {subgroup }}}$ $A_{1}$ of order 2. Clearly, $A_{1} \subseteq \mathbf{O}_{\theta}\left(\mathcal{B}_{G / P}\right)$. In view of Equation (9.6), we have $A_{1} \neq A$. Thus, in both cases $\mathbf{O}_{\theta}\left(\mathcal{B}_{G / P}\right) \not A$. Together with Equation (9.3) this implies that

$$
\left|\mathbf{O}_{\theta}\left(\mathcal{B}_{G / P}\right)\right| \geq 4 \text {. }
$$

If $\mathcal{B}_{G / P}$ is indecomposable then $\mathcal{B}_{G / P}$ is normal by Lemma 8.3 and hence $\mathcal{B}$ is $C$ I by Lemma 9.2 and Lemma 5.5. If $S$ has a gwr-complement with respect to $\mathcal{B}_{G / P}$ then $\mathcal{B}$ is CI by Lemma 9.4. If $\mathbf{O}_{\theta}\left(\mathcal{B}_{G / P}\right) \not \leq S$ then $\mathcal{B}$ is CI by Lemma 9.2 and Lemma 5.8. Suppose that none of the above conditions does not hold for $\mathcal{B}$. Then, in view of Equation (9.7), $\mathcal{B}$ satisfies all conditions from one of the Cases 1,2 or 3 . Therefore, $\mathcal{B}$ is CI.

The $S$-ring $\mathcal{A}_{G / L}$ is normal because it is isomorphic to $\mathcal{A}_{(G / P) / B}$. The $S$-rings $\mathcal{A}_{G / L}$ and $\mathcal{B}$ are CI by Lemma 9.2 and the above paragraph respectively. Thus, $\mathcal{A}$ is CI by Lemma 4.4 .

All cases were considered.

Proof of Theorem 9.1. Let $H_{1}$ be a maximal $\mathcal{A}$-subgroup contained in $H$ and $P_{1}$ the least $\mathcal{A}$-subgroup containing $P$.

Lemma 9.6. If $H_{1}=H$ then $\mathcal{A}$ is a CI-S-ring.

Proof. The $S$-ring $\mathcal{A}_{G / H}$ is a $p$ - $S$-ring over $G / H \cong C_{p}$ by Lemma 6.4. So $\mathcal{A}_{G / H} \cong \mathbb{Z} C_{p}$. Clearly, $G=H P_{1}$. Therefore $\mathcal{A}=\mathcal{A}_{H} \star \mathcal{A}_{P_{1}}$ by Lemma 7.2. Since $H$ and $P_{1} /\left(H \cap P_{1}\right)$ are proper sections of $G$, the $S$-rings $\mathcal{A}_{H}$ and $\mathcal{A}_{P_{1} /\left(H \cap P_{1}\right)}$ are CI by Lemma 9.2. Thus, $\mathcal{A}$ is CI by Lemma 5.6.

Lemma 9.7. If $H_{1}<H$ and $H_{1} P_{1}=G$ then $\mathcal{A}$ is a CI- $S$-ring. 
Proof. Since $H_{1} \neq\left(H_{1} P_{1}\right)_{p^{\prime}}=H$, Lemma 7.1 implies that $\mathcal{A}=\mathcal{A}_{H_{1}} \star \mathcal{A}_{P_{1}}$. The $S$-rings $\mathcal{A}_{H_{1}}$ and $\mathcal{A}_{P_{1} /\left(H \cap P_{1}\right)}$ are CI by Lemma 9.2 because $H_{1}$ and $P_{1} /\left(H_{1} \cap P_{1}\right)$ are proper sections of $G$. Therefore $\mathcal{A}$ is CI by Lemma 5.6.

In view of Lemma 9.6, we may assume that $H_{1}<H$. Then one of the statements of Lemma 7.3 holds for $\mathcal{A}$. If Statement (1) of Lemma 7.3 holds for $\mathcal{A}$ then

$$
\mathcal{A}=\mathcal{A}_{H_{1}} \prec \mathcal{A}_{G / H_{1}},
$$

where $\operatorname{rk}\left(\mathcal{A}_{G / H_{1}}\right)=2$. If $H_{1}$ is trivial then $\operatorname{rk}(\mathcal{A})=2$. Obviously, $\mathcal{A}$ is $\mathrm{CI}$ in this case. If $H_{1}$ is nontrivial then $\mathcal{A}$ is CI by Lemma 9.2 and Lemma 5.2.

Assume that Statement (2) of Lemma 7.3 holds for $\mathcal{A}$, i.e.

$$
\mathcal{A}=\mathcal{A}_{U}{ }_{{ }_{S}} \mathcal{A}_{G / P_{1}},
$$

where $U=H_{1} P_{1}, S=U / P_{1}$, and $P_{1}<G$. In view of Lemma 9.7, we may assume that $H_{1} P_{1}<G$, i.e. $\mathcal{A}$ is the nontrivial $S$-wreath product. The group $G / P_{1}$ is a 2 -group of order at most 32 because $P_{1} \geq P$. Lemma 6.4 implies that $\mathcal{A}_{G / P_{1}}$ is a $2-S$-ring. If $\left|G / P_{1}\right| \leq 16$ then $\mathcal{A}$ is CI by Lemma 9.2 and Lemma 8.9. So we may assume that $\left|G / P_{1}\right|=32$. Clearly, in this case

$$
P_{1}=P
$$

In view of Statement (2) of Lemma 6.3, we may assume that

$$
|S|=16 .
$$

Indeed, if $|S|<16$ then $S$ is contained in an $\mathcal{A}_{G / P}$-subgroup $S^{\prime}$ of order 16 by Statement (2) of Lemma 6.3. Clearly, $\mathcal{A}=\mathcal{A}_{U^{\prime}}{ }_{S_{S}} \mathcal{A}_{G / P}$, where $U^{\prime}=\pi^{-1}\left(S^{\prime}\right)$ and $\pi: G \rightarrow$ $G / P$ is the canonical epimorphism. Replacing $S$ by $S^{\prime}$, we obtain the required.

Now all conditions of Lemma 9.3 hold for $\mathcal{A}$ and $U$. Thus, $\mathcal{A}$ is CI by Lemma 9.3.

\section{ORCID iD}

Grigory Ryabov (iD https://orcid.org/0000-0001-5935-141X

\section{References}

[1] A. Ádám, Research problem 2-10, J. Comb. Theory 2 (1967), 393, doi:10.1016/s0021-9800(67) 80037-1.

[2] B. Alspach and L. A. Nowitz, Elementary proofs that $Z_{p}^{2}$ and $Z_{p}^{3}$ are CI-groups, European J. Combin. 20 (1999), 607-617, doi:10.1006/eujc.1999.0309.

[3] B. Alspach and T. D. Parsons, Isomorphism of circulant graphs and digraphs, Discrete Math. 25 (1979), 97-108, doi:10.1016/0012-365x(79)90011-6.

[4] L. Babai, Isomorphism problem for a class of point-symmetric structures, Acta Math. Acad. Sci. Hungar. 29 (1977), 329-336, doi:10.1007/bf01895854.

[5] L. Babai and P. Frankl, Isomorphisms of Cayley graphs I, in: A. Hajnal and V. T. Sós (eds.), Combinatorics, Volume I, North-Holland, Amsterdam-New York, volume 18 of Colloquia Mathematica Societatis János Bolyai, 1978 pp. 35-52, proceedings of the 5th Hungarian Colloquium held at Keszthely, June 28 - July 3, 1976. 
[6] G. Chen and I. Ponomarenko, Lectures on Coherent Configurations, Central China Normal University Press, Wuhan, 2019, http://www.pdmi.ras.ru/ inp/ccNOTES.pdf.

[7] M. Conder and C. H. Li, On isomorphisms of finite Cayley graphs, European J. Combin. 19 (1998), 911-919, doi:10.1006/eujc.1998.0243.

[8] D. Ž. Djoković, Isomorphism problem for a special class of graphs, Acta Math. Acad. Sci. Hungar. 21 (1970), 267-270, doi:10.1007/bf01894773.

[9] E. Dobson, Isomorphism problem for Cayley graphs of $Z_{p}^{3}$, Discrete Math. 147 (1995), 87-94, doi:10.1016/0012-365x(95)00099-i.

[10] B. Elspas and J. Turner, Graphs with circulant adjacency matrices, J. Comb. Theory 9 (1970), 297-307, doi:10.1016/s0021-9800(70)80068-0.

[11] S. Evdokimov, I. Kovács and I. Ponomarenko, On schurity of finite abelian groups, Comm. Algebra 44 (2016), 101-117, doi:10.1080/00927872.2014.958848.

[12] S. Evdokimov and I. Ponomarenko, On a family of Schur rings over a finite cyclic group, St. Petersburg Math. J. 12 (2002), 441-451, http: / / mi . mathnet.ru/eng/aa 940.

[13] Y.-Q. Feng and I. Kovács, Elementary abelian groups of rank 5 are DCI-groups, J. Comb. Theory Ser. A 157 (2018), 162-204, doi:10.1016/j.jcta.2018.02.003.

[14] C. D. Godsil, On Cayley graph isomorphisms, Ars Combin. 15 (1983), 231-246.

[15] M. Hirasaka and M. Muzychuk, An elementary abelian group of rank 4 is a CI-group, J. Comb. Theory Ser. A 94 (2001), 339-362, doi:10.1006/jcta.2000.3140.

[16] M. Klin, C. Pech and S. Reichard, COCO2P - a GAP package, Version 0.14, 7 February 2015, https://github.com/chpech/COCO2P.

[17] M. H. Klin and R. Pöschel, The König problem, the isomorphism problem for cyclic graphs and the method of Schur rings, in: L. Lovász and V. T. Sós (eds.), Algebraic Methods in Graph Theory, Volume I, North-Holland, Amsterdam-New York, volume 25 of Colloquia Mathematica Societatis János Bolyai, pp. 405-434, 1981, papers from the Conference held in Szeged, August 24 - 31, 1978.

[18] I. Kovács and M. Muzychuk, The group $\mathbb{Z}_{p}^{2} \times \mathbb{Z}_{q}$ is a CI-group, Comm. Algebra 37 (2009), 3500-3515, doi:10.1080/00927870802504957.

[19] I. Kovács and G. Ryabov, $C I$-property for decomposable Schur rings over an abelian group, Algebra Colloq. 26 (2019), 147-160, doi:10.1142/s1005386719000142.

[20] I. Kovács and G. Ryabov, The group $C_{p}^{4} \times C_{q}$ is a DCI-group, 2019, arXiv:1912.08835 [math.CO].

[21] C. H. Li, On isomorphisms of finite Cayley graphs—a survey, Discrete Math. 256 (2002), 301-334, doi:10.1016/s0012-365x(01)00438-1.

[22] J. Morris, Elementary proof that $\mathbb{Z}_{p}^{4}$ is a DCI-group, Discrete Math. 338 (2015), 1385-1393, doi:10.1016/j.disc.2015.02.023.

[23] M. Muzychuk, Ádám's conjecture is true in the square-free case, J. Comb. Theory Ser. A 72 (1995), 118-134, doi:10.1016/0097-3165(95)90031-4.

[24] M. Muzychuk, On Ádám's conjecture for circulant graphs, Discrete Math. 167/168 (1997), 497-510, doi:10.1016/s0012-365x(96)00251-8.

[25] M. Muzychuk and I. Ponomarenko, Schur rings, European J. Combin. 30 (2009), 1526-1539, doi:10.1016/j.ejc.2008.11.006.

[26] M. Muzychuk and I. Ponomarenko, On Schur 2-groups, Zap. Nauchn. Sem. S.-Peterburg. Otdel. Mat. Inst. Steklov. (POMI) 435 (2015), 113-162, doi:10.1007/s10958-016-3128-z. 
[27] M. Muzychuk and G. Somlai, The Cayley isomorphism property for $\mathbb{Z}_{p}^{3} \times \mathbb{Z}_{q}, 2019$, arXiv:1907.03570 [math.GR].

[28] L. A. Nowitz, A non-Cayley-invariant Cayley graph of the elementary abelian group of order 64, Discrete Math. 110 (1992), 223-228, doi:10.1016/0012-365x(92)90711-n.

[29] I. N. Ponomarenko and A. Rakhnama Bargi, On the structure of p-schemes, Zap. Nauchn. Sem. S.-Peterburg. Otdel. Mat. Inst. Steklov. (POMI) 344 (2007), 190-202, doi:10.1007/ s10958-007-0539-x.

[30] S. Reichard, Schur rings over small groups, http://www.math.tu-dresden.de/ reichard/schur/newData/.

[31] I. Schur, Zur Theorie der einfach transitiven Permutationsgruppen, Sitzungsber. Preuß. Akad. Wiss., Phys.-Math. Kl. 18 (1933), 598-623.

[32] G. Somlai, Elementary abelian $p$-groups of rank $2 p+3$ are not CI-groups, J. Algebraic Combin. 34 (2011), 323-335, doi:10.1007/s10801-011-0273-9.

[33] P. Spiga, CI-property of elementary abelian 3-groups, Discrete Math. 309 (2009), 3393-3398, doi:10.1016/j.disc.2008.08.002.

[34] H. Wielandt, Finite Permutation Groups, Academic Press, London, 1964, doi:10.1016/ c2013-0-11702-3.

[35] M. Ziv-Av, Enumeration of Schur rings over small groups, in: V. P. Gerdt, W. Koepf, W. M. Seiler and E. V. Vorozhtsov (eds.), Computer Algebra in Scientific Computing, Springer, Cham, volume 8660 of Lecture Notes in Computer Science, 2014 pp. 491-500, doi:10.1007/ 978-3-319-10515-4_35, proceedings of the 16th International Workshop (CASC 2014) held in Warsaw, September $8-12,2014$. 